\title{
MULTIAMICABLE NUMBERS
}

GRAEME L. COHEN, STEPHEN F. GRETTON, AND PETER HAGIS, JR.

\begin{abstract}
Multiamicable numbers are a natural generalization of amicable numbers: two numbers form a multiamicable pair if the sum of the proper divisors of each is a multiple of the other. Many other generalizations have been considered in the past. This paper reviews those earlier generalizations and gives examples and properties of multiamicable pairs. It includes a proof that the set of all multiamicable numbers has density 0 .
\end{abstract}

\section{Generalizations of amicable Numbers}

Two natural numbers are said to be amicable if the sum of the proper divisors of each of them equals the other. Thus, where $\sigma$ denotes the positive divisor sum function, $m$ and $n$ are amicable if

$$
\sigma(m)-m=n \quad \text { and } \quad \sigma(n)-n=m .
$$

This condition can be abbreviated to $\sigma(m)=\sigma(n)=m+n$. We assume that $m \neq n$. (If $m=n$, then $m$ is perfect.) It is usual to order pairs of amicable numbers according to the size of the smaller member. The smallest pair of amicable numbers, known to the Pythagoreans, is 220 and 284. Many of the classical mathematicians, such as Fermat, Mersenne, Descartes, Legendre, and particularly Euler, studied amicable numbers. Over fifty thousand pairs are now known, and many techniques are known for generating new pairs from old ones, although these will not always be successful. It has not been determined, however, whether there are infinitely many pairs of amicable numbers. For a discussion of these and for further references, see Battiato and Borho [1], Guy [10] and te Riele [18].

A number of generalizations appear in the literature.

Our first, due to Dickson [6], defines $k$ natural numbers $n_{1}, n_{2}, \ldots, n_{k}$ to be an amicable $k$-tuple if

$$
\sigma\left(n_{i}\right)=\sum_{j=1}^{k} n_{j} \quad \text { for } i=1, \ldots, k,
$$

that is, if the sum of the proper divisors of each equals the sum of all the others. Dickson gave ten examples of amicable triples (not counting examples in which all $n_{i}$ are equal).

Received by the editor December 14, 1992 and, in revised form, March 22, 1994 and August 23, 1994.

1991 Mathematics Subject Classification. Primary 11A25.

The research for this paper was completed while the first author was a Visiting Research Associate at Temple University, Philadelphia. 
Mason [14] applied Euler's approach for finding amicable pairs to Dickson's definition in order to generate more amicable (pairs and) triples, in addition to 14 amicable quadruples, three amicable quintuples and seven amicable sextuples. (More could now be quickly obtained. The method makes use of the current list of multiperfect numbers with a certain property. These will be defined later.) Poulet [17] also spoke of "nombres multiamiables". These were $k$-tuples satisfying Dickson's definition. He gave 147 "nombres triamiables", as well as many amicable quadruples, quintuples and sextuples, including all those of Dickson and Mason, with whose work he was clearly familiar. Much later, Makowski [13] found two smaller examples of amicable triples: $2^{2} 3^{2} 5 \cdot 11$, $2^{5} 3^{2} 7,2^{2} 3^{2} 71$ and $2^{3} 3 \cdot 5 \cdot 13,2^{2} 3 \cdot 5 \cdot 29,2^{2} 3 \cdot 5 \cdot 29$. Poulet had missed these.

Yanney [20] defined the numbers $n_{1}, n_{2}, \ldots, n_{k}$ to be an amicable $k$-tuple (that is, using the same symbolism and name) when each equals the sum of the proper divisors of all the others. This can be shown to be equivalent to the requirement that

$$
(k-1) \sigma\left(n_{i}\right)=\sum_{j=1}^{k} n_{j} \quad \text { for } i=1, \ldots, k .
$$

(In fact, as Yanney noted, E. B. Escott had asked much. earlier whether such numbers exist; see Dickson [7, page 50].) There are trivial examples, such as 2 , $2,2(k=3)$ and $3,3,3,3(k=4)$. Yanney also gave the example $2^{2} 7 \cdot 11$, $5 \cdot 7 \cdot 13,7 \cdot 83$.

Both of these definitions revert to that of an amicable pair, as originally defined, when $k=2$. Yanney related his definition to that of Dickson, and gave a further generalization which includes both definitions.

In Mason's paper, mentioned above, he also gave many examples of a further extension of Dickson's definition, due to Carmichael [2]: the numbers $n_{1}, n_{2}$, $\ldots, n_{k}$ are said to be a multiply amicable $k$-tuple if

$$
\sigma\left(n_{i}\right)=t \sum_{j=1}^{k} n_{j} \quad \text { for } i=1, \ldots, k,
$$

where $t$ is an integer. He deduced a table of factors by use of which "in connection with a table of the known multiply perfect [or multiperfect] numbers about six hundred multiply amicable number pairs can be obtained of multiplicity $t=2,3$ ". Presumably, many hundreds more multiply amicable numbers can now be found, including some with multiplicity $t=4$. Other examples, derived independently of the table, are also given.

The most investigated generalizations of amicable numbers are the aliquot cycles or sociable numbers. These are numbers $n_{1}, n_{2}, \ldots, n_{k}$ with the property that $\sigma\left(n_{1}\right)-n_{1}=n_{2}, \sigma\left(n_{2}\right)-n_{2}=n_{3}, \ldots, \sigma\left(n_{k-1}\right)-n_{k-1}=n_{k}$, $\sigma\left(n_{k}\right)-n_{k}=n_{1}$. When $k=1$, we have a perfect number; when $k=2$, we have an amicable pair. No examples are known with $k=3$, but cycles with periods 4, 5, 8, 9 and 28 have been found. See Flammenkamp [9], Guy [10] and Moews and Moews [16].

There have also been numerous other investigations where the notion of amicable numbers has been varied, rather than generalized. Either a different definition of "divisor" has been adopted, as with unitary amicable numbers (see 
Hagis [11]) or infinitary amicable numbers (see Cohen [3]), or the conditions in (1) have been altered slightly. For the latter, see Guy [10] where pairs $m, n$ with $\sigma(m)=\sigma(n)=m+n-1$ or with $\sigma(m)=\sigma(n)=m+n+1$ are discussed.

See also Dickson [7, page 50], Krishnamurthy [12] and Sierpiñski [19, page 187].

\section{Multiamicable NUmbers}

Multiamicable numbers are a natural generalization of amicable numbers, coming about in precisely the same fashion as the definition of multiperfect numbers generalizes that of perfect numbers.

Traditionally, a number is perfect if the sum of its proper divisors equals the number; and a number is multiperfect if the sum of its proper divisors is a multiple of the number. Thus, $n$ is $\gamma$-fold multiply perfect if $\sigma(n)-n=$ $\gamma n$. (Certainly, this is the approach of McDaniel [15] and others. However, Dickson [7, page 33], writes: "A multiply perfect or pluperfect number $n$ is one the sum of whose divisors, including $n$ and 1 , is a multiple of $n$. If the sum is $m n, m$ is called the multiplicity of $n$.") Since we have $\sigma(n)=(\gamma+1) n$, by a natural corruption of the original intent, such $n$ became known as $(\gamma+1)$ perfect.

We define natural numbers $m$ and $n$ to be multiamicable if the sum of the proper divisors of each of them is a multiple of the other. More precisely, we shall call $m$ and $n(\alpha, \beta)$-amicable if

$$
\sigma(m)-m=\alpha n \quad \text { and } \quad \sigma(n)-n=\beta m .
$$

Of course, $\alpha$ and $\beta$ must be positive integers, and if $\alpha=\beta=1$, then $m$ and $n$ are amicable. By a proper $(\alpha, \beta)$-amicable pair, we shall mean one with $\alpha \beta>1$.

There is no extra interest in allowing the possibility that $m=n$, so we shall assume always that $m<n$, with the pair $(\alpha, \beta)$ correspondingly ordered. Then

$$
(\alpha+1) m<\sigma(m)<(\alpha+1) n \quad \text { and } \quad(\beta+1) m<\sigma(n)<(\beta+1) n ;
$$

in particular, the left-most inequality states that $m$ is $(\alpha+1)$-abundant. This quickly implies some restrictions on $m$ or $\alpha$. For example, $m$ cannot have just one distinct prime factor, and if it has precisely two distinct prime factors, then $\alpha=1$ and $m$ is even. To prove the second of these remarks, suppose $m=p^{a} q^{b}$ is the prime factorization of $m$. Then

$$
\alpha+1<\frac{\sigma(m)}{m}<\frac{p}{p-1} \cdot \frac{q}{q-1} \leq \frac{2}{1} \cdot \frac{3}{2}=3,
$$

so $\alpha=1$; the assumption $3 \leq p<q$ would lead to a contradiction.

A search by brute force revealed the three $(1,7)$-amicable pairs

$$
m=52920=2^{3} 3^{3} 5 \cdot 7^{2}, \quad n=152280=2^{3} 3^{4} 5 \cdot 47,
$$

(II)

$$
m=16225560=2^{3} 3^{2} 5 \cdot 13 \cdot 3467, \quad n=40580280=2^{3} 3^{2} 5 \cdot 13^{2} 23 \cdot 29,
$$

$$
m=90863136=2^{5} 3^{2} 7 \cdot 13 \cdot 3467, \quad n=227249568=2^{5} 3^{2} 7 \cdot 13^{2} 23 \cdot 29,
$$


the two $(2,5)$-amicable pairs

$$
m=3898440=2^{3} 3^{2} 5 \cdot 7^{2} 13 \cdot 17, \quad n=6453720=2^{3} 3^{2} 5 \cdot 7 \cdot 13 \cdot 197,
$$

and the $(1,6)$-amicable pair

(VI) $m=76455288=2^{3} 3^{2} 7^{2} 13 \cdot 1667, \quad n=183102192=2^{4} 3^{2} 7 \cdot 13 \cdot 89 \cdot 157$.

This comprehensive search showed that there are no other proper $(\alpha, \beta)$ amicable pairs $m, n$ with $m \leq 10^{8}$. The search incorporated the fact that $\alpha \leq 4$ for any such pair. To see this, suppose $\alpha \geq 5$. Then $m$ must have at least nine distinct prime factors, since otherwise, using (3),

$$
6 \leq \alpha+1<\frac{\sigma(m)}{m}<2 \cdot \frac{3}{2} \cdot \frac{5}{4} \cdot \frac{7}{6} \cdot \frac{11}{10} \cdot \frac{13}{12} \cdot \frac{17}{16} \cdot \frac{19}{18}<6 ;
$$

but then $m>2 \cdot 3 \cdot 5 \cdot 7 \cdot 11 \cdot 13 \cdot 17 \cdot 19 \cdot 23>10^{8}$. (We note that H. Cohen [5] determined that there are 236 ordinary amicable pairs with smaller member less than $10^{8}$.)

The factorizations of $m$ and $n$ in the preceding examples led to the following proposition, which in turn led to the others of the 78 known multiamicable pairs, listed in Table 1.

Proposition 1. Suppose the natural numbers $M, N, \alpha, \quad \beta$ and a satisfy $\operatorname{gcd}(a, M)=\operatorname{gcd}(a, N)=1$ and

$$
\frac{\sigma(a)}{a}=\frac{M+\alpha N}{\sigma(M)}=\frac{\beta M+N}{\sigma(N)} .
$$

Then aM, aN are an $(\alpha, \beta)$-amicable pair.

This follows easily using the multiplicativity of $\sigma$.

To illustrate its use, observe from example (I) that we may take $\alpha=1$, $\beta=7, a=2^{3} 5$, and, for $X$ and $Y$ in

$$
\frac{X+\alpha Y}{\sigma(X)}=\frac{\beta X+Y}{\sigma(Y)}
$$

$X=M=3^{3} 7^{2}$ and $Y=N=3^{4} 47$. Here, $\sigma(a) / a=9 / 4$. We will obtain further (1,7)-pairs from other values of $a$ satisfying $\sigma(a) / a=9 / 4=A$, say, provided also $\operatorname{gcd}(a, M)=\operatorname{gcd}(a, N)=1$. We adopted the following approach to finding such values of $a$. Seek those $a$ of the form $b / p$, where $\sigma(b)=\delta b$ for some $\delta$ (not necessarily an integer), and where $p$ is a prime exactly dividing $b$. For such $a$,

$$
A=\frac{\sigma(b / p)}{b / p}=\frac{\sigma(b)}{p+1} \cdot \frac{p}{b}=\frac{\delta p}{p+1},
$$

so that we require $\delta=A(p+1) / p$. In our example, $\delta=9(p+1) / 4 p$. Clearly, we should take $p=3$, so that $\delta=3$, and then for $b$ we need 3-perfect numbers exactly divisible by 3 . Four such numbers are known: $2^{3} 3 \cdot 5,2^{5} 3 \cdot 7$, $2^{9} 3 \cdot 11 \cdot 31$ and $2^{13} 3 \cdot 11 \cdot 43 \cdot 127$. If $b$ is any of these, take $a=b / 3$, provided such $a$ is relatively prime to $M$ and $N$. Three suitable values remain for $a$. The first is our original example (I); all three are given in line (ii) of Table 1. 
TABLE 1. All known multiamicable pairs; the numbers $a M$ and $a N$

\begin{tabular}{|c|c|c|c|c|c|c|c|}
\hline & $\alpha$ & $\beta$ & $M$ & $N$ & $\frac{\sigma(a)}{a}$ & $a$ & No \\
\hline (i) & 1 & 6 & $2^{3} 7^{2} 1667$ & $2^{4} 7 \cdot 89 \cdot 157$ & $\frac{14}{9}$ & $3^{2} 13$ & 1 \\
\hline (ii) & 1 & 7 & $3^{3} 7^{2}$ & $3^{4} 47$ & $\frac{9}{4}$ & $\frac{C x}{3}, \quad x=1,4,5$ & 3 \\
\hline (iii) & 1 & 7 & $13 \cdot 3467$ & $13^{2} 23 \cdot 29$ & $\frac{13}{4}$ & $\frac{D x}{7 \cdot 13}, \quad x=2,12,16$ & \\
\hline & & & & & & $2^{5} 3^{2} 7$ & 4 \\
\hline (iv) & 1 & 10 & 3 & $3^{2}$ & 3 & $C x, \quad x=3,6$ & 2 \\
\hline (v) & 1 & 26 & 5 & $5^{2}$ & 5 & $\begin{array}{c}E x, \\
x=29,30,36,37,38,39 \\
40,41,45,50,51,52,53 \\
54,55,56,57,58,59,60\end{array}$ & 20 \\
\hline (vi) & 2 & 4 & $3^{3}$ & $3 \cdot 13$ & $\frac{21}{8}$ & $\frac{C x}{7}, \quad x=3,6$ & 2 \\
\hline (vii) & 2 & 5 & $7^{2} 17$ & $7 \cdot 197$ & $\frac{7}{2}$ & $\begin{array}{c}\frac{D x}{7}, \\
x=2,3,9,12,14,16,18 \\
20,21,22,23,25,26 \\
31,32,33,34,35,36\end{array}$ & 19 \\
\hline (viii) & 3 & 5 & 137 & $13^{2}$ & $\frac{14}{3}$ & $\begin{array}{c}\text { solutions found by S. Gretton; } \\
\text { see Table } 2\end{array}$ & 14 \\
\hline $\begin{array}{r}(\mathbf{i x}) \\
(\mathrm{x})\end{array}$ & $\begin{array}{l}3 \\
3\end{array}$ & $\begin{array}{l}7 \\
7\end{array}$ & $\begin{array}{c}7 \\
67\end{array}$ & $\begin{array}{c}11 \\
7 \cdot 13\end{array}$ & $\begin{array}{l}5 \\
5\end{array}$ & $\begin{array}{c}E 46 \\
E x, \\
x=31,32,33,61,62,63,64\end{array}$ & $\begin{array}{l}1 \\
7\end{array}$ \\
\hline (xi) & 3 & 16 & 59 & 137 & $\frac{47}{6}$ & $\frac{H x}{47}, \quad x=2,3,6,11,12$ & 5 \\
\hline
\end{tabular}

Examples (IV) and (V) led similarly to line (vii) of Table 1. Examples (II) and (III) are a little more complicated, and will be discussed below. The $(1,6)$ pair of example (VI) is repeated in line (i) of Table 1; it is our only example with different powers of 2 in $m$ and $n$.

In applying Proposition 1, we made extensive use of the list of all known multiperfect numbers recently compiled by Rich Schroeppel. Our list is labelled "mpfn731", and dated 4 November 1992. Schroeppel refers to multiperfect numbers as MPFNs, and there are 731 numbers in the list; hence the label. The numbers have multiplicities from 1 (for the trivial example 1) through 8 ; the 32 known even perfect numbers are included. The list is available by electronic mail at rcs@cs.arizona.edu. ${ }^{1}$

${ }^{1}$ Added March 1994: Many more multiperfect numbers have since been found, including some of multiplicity 9 and the 33rd even perfect number. At this time, almost 1800 multiperfect numbers are known, and certainly many more multiamicable pairs can be identified. A complete list, at the time, of 1385 multiperfect numbers was compiled and made available by Schroeppel in May 1993. The situation at present is very dynamic, and until it settles down we will not attempt any further comprehensive listing of multiamicable pairs. 
In Table 1 , the notation $X x$ refers to the numbering system which Schroeppel has adopted for his list of multiperfect numbers (and which may well alter as subsequent multiperfect numbers are discovered and later lists are produced); $X$ denotes the index $(B, C, \ldots, H$ for 2-perfect, 3-perfect, $\ldots$, 8-perfect numbers) and $x$ the number of the multiperfect number with that index. Referring again to our example, $C 1, C 4$ and $C 5$ are the first, third and fourth of the 3-perfect numbers given above.

Proposition 1 was also used in the following manner. Solutions of (4) are easily generated by computer. We determined all solutions $M$ and $N$ (for $X$ and $Y$, respectively) with $1 \leq M<945$ and $M<N<945$, subject also to some practical constraints to be described, and we then sought corresponding values for $a$, where $\sigma(a) / a=(M+\alpha N) / \sigma(M)=A$, say. The earlier approach and Schroeppel's lists were again used for finding values of $a$. Since all numbers in the lists (except for the trivial 1) are even, we only considered odd values for $M$ and $N$, and since 8 is the maximum multiplicity for numbers in the lists, and $A=\delta p /(p+1)$, we only considered those solutions with $A<8$. These constraints limit the possible values of $\alpha$ and $\beta$. For the smallest odd 2-abundant number is 945 , so $\sigma(M)<2 M$ and $\sigma(N)<2 N$ for any solutions in our range, and then

$$
8>A=\frac{M+\alpha N}{\sigma(M)}>\frac{M+\alpha N}{2 M}
$$

from which $\alpha<15 M / N$, and similarly $\beta<15 N / M$. We also required $\alpha \beta>1$.

Furthermore, we restricted our attention to solutions $M$ and $N$ with greatest common unitary divisor equal to 1 (and we termed these primitive solutions), since we were able to show that if a primitive solution $M, N$ led to an $(\alpha, \beta)$ amicable pair, then any solution $\lambda M, \lambda N$, where $\operatorname{gcd}(\lambda, M)=\operatorname{gcd}(\lambda, N)=1$, would lead at best to another $(\alpha, \beta)$-amicable pair corresponding to the solution $M, N$. (Note: a divisor $d$ of $l$ is unitary if $\operatorname{gcd}(d, l / d)=1$. )

In one instance, arising from example (II) above, an iterative use was made of Table 1 . That example required values of $a$ satisfying $\sigma(a) / a=13 / 4$. The approach described above led to $p=13$ and $\delta=7 / 2$, and line (vii) of Table 1 gives solutions of $\sigma(b) / b=7 / 2$. The resulting pairs are in line (iii). This approach cannot find values of $a$ divisible by 7 . We have seen such a value; it occurred in example (III) and is repeated within line (iii).

In another instance, requiring the solution of $\sigma(a) / a=14 / 3$, no multiamicable pairs can be generated in the manner described above. However, by means similar to those by which multiperfect numbers are found, fourteen admissible solutions of this equation were obtained, and the resulting multiamicable pairs are described in line (viii) of Table 1 and listed in Table 2.

There were 61 solutions of (4) which satisfied our constraints and only seven of these led us to multiamicable pairs. It is interesting that the only one of the 61 primitive solutions for which $\alpha \geq \beta$ (and $\alpha \beta>1$ ) was $\alpha=8, \beta=7$, $X=3^{3}, Y=31$.

It will be noticed from Table 1 that there are 22 known examples of multiamicable pairs $m, n$ in which $m \mid n$. We can show by different means that there 
TABLE 2. (3, 5)-amicable pairs

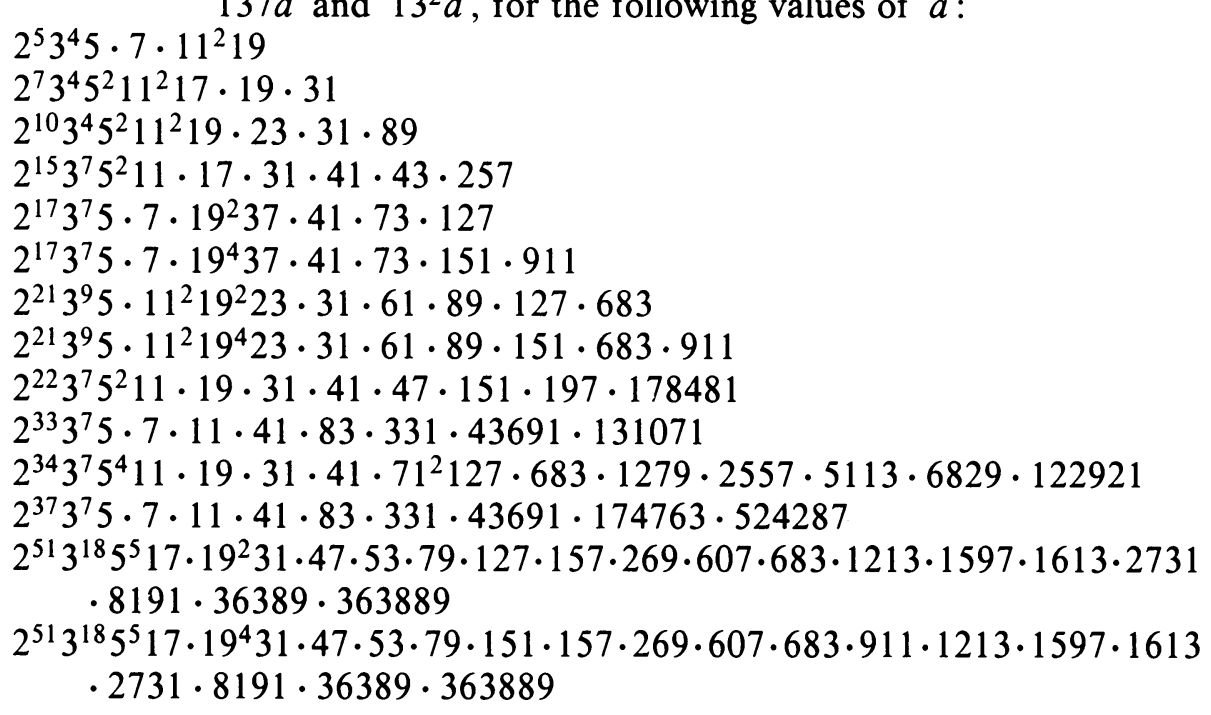

are no other such examples, given today's knowledge of multiperfect numbers. (See Cohen [4]. This remark is subject also to the comment in the footnote above.)

One important difference between our multiamicable pairs and Carmichael's multiply amicable pairs, defined in (2) with $k=2$, is that the latter definition requires $\sigma(m)=\sigma(n)$. In fact, all the other generalizations of amicable numbers, other than that to sociable numbers, maintained such a requirement. Many of the techniques for finding amicable $k$-tuples and multiply amicable sets of numbers made strong use of this as the first step.

We cannot rule out the existence of multiamicable pairs $m, n$ with $\sigma(m)=$ $\sigma(n)$. The next proposition implies that any such pairs must be very large.

Proposition 2. If $m, n$ are a proper $(\alpha, \beta)$-amicable pair and $\sigma(m)=\sigma(n)$, then $7 \leq \alpha<\beta$ and $\sigma(m) / m>\beta+1$.

Proof. We have $m+\alpha n=\sigma(m)=\sigma(n)=\beta m+n$, so that

$$
(\alpha-1) n=(\beta-1) m \text {. }
$$

We cannot have $\alpha=1$, since then $\beta=1$; but $\alpha \beta>1$. Since $m<n$, then (5) implies that $\alpha<\beta$. If $\alpha=2$ then $m \mid n$, which is impossible when $\sigma(m)=\sigma(n)$ and $m \neq n$. Observe next that, for positive integers $r$ and $s$,

$$
\sigma(r) \sigma(s) \geq \sigma(r s)>r \sigma(s) \text { when } r>1,
$$

so, using this and (5), we get

$$
\sigma(\alpha-1) \sigma(n) \geq \sigma((\alpha-1) n)=\sigma((\beta-1) m)>(\beta-1) \sigma(m) .
$$

We have $\sigma(m)=\sigma(n)$ and $\beta \geq \alpha+1$, so, when $\alpha-1$ is prime,

$$
\alpha=\sigma(\alpha-1)>\beta-1 \geq \alpha .
$$

This contradiction shows that $\alpha \neq 3,4$ or 6 . 
Now suppose that $\alpha=5$, so that $\beta \geq 6$. From (5), $4 n=(\beta-1) m$. Write $n=2^{a} l$, where $l$ is odd. Then

$$
\sigma\left(2^{a+2}\right) \sigma(l)=\sigma(4 n)=\sigma((\beta-1) m)
$$

and $\sigma(n)=\sigma\left(2^{a}\right) \sigma(l)$, so, by $(6)$,

$$
\frac{\sigma\left(2^{a+2}\right)}{\sigma\left(2^{a}\right)} \sigma(n)=\sigma((\beta-1) m)>(\beta-1) \sigma(m) .
$$

Hence, since $\sigma(m)=\sigma(n)$,

$$
\beta<1+\frac{2^{a+3}-1}{2^{a+1}-1}=5+\frac{3}{2^{a+1}-1} .
$$

Since $\beta \geq 6$, we thus have $a=0$, and then $\beta<8$. There are two cases to consider.

(1) If $\beta=6$, then, using (6) and the fact that $n$ is odd, we are led to

$$
7 \sigma(n)=\sigma(4 n)=\sigma((\beta-1) m)=\sigma(5 m) \leq \sigma(5) \sigma(m)=6 \sigma(m),
$$

and we have a contradiction.

(2) If $\beta=7$, then, since $2 n=3 m$, we know $3 \mid n$. Write $n=3^{b} k$, where $k$ is not divisible by 3 . Then $2 \cdot 3^{b-1} k=m$ and $k$ is odd, so

$$
3 \sigma\left(3^{b-1}\right) \sigma(k)=\sigma(m)=\sigma(n)=\sigma\left(3^{b}\right) \sigma(k) .
$$

We cannot have $3 \sigma\left(3^{b-1}\right)=\sigma\left(3^{b}\right)$ for any $b$, so again we have a contradiction.

This proves that $\alpha \geq 7$. Note finally that, using (5) and the fact that $\beta>$ $\alpha>1$, we have

$$
\sigma(m)=m+\alpha n=m+\alpha \frac{\beta-1}{\alpha-1} m=\frac{\alpha \beta-1}{\alpha-1} m>(\beta+1) m .
$$

Two interesting observations arise from this proof. First, the right-hand inequality in (6) is equivalent to $\sigma(r s) / r s>\sigma(s) / s(r>1)$, which is a restatement of the following familiar result in this area of study: if $u$ is a proper divisor of $v$, then $\sigma(u) / u<\sigma(v) / v$. Secondly, we proved in passing the general result that if $\sigma(m)=\sigma(n)$ (where $m<n$ ) and we write $x m=y n$ for positive integers $x$ and $y$, then $y \geq 6$ (and $y$ cannot be prime). The fact that $\sigma(6)=\sigma(11)$ shows that this is best possible.

We end with a result concerning the density of multiamicable numbers. In [8], Erdős proved that amicable numbers have density 0 . His proof rested on the following lemma: For a fixed integer $N>0$, the set of integers $l$ for which $N$ does not divide $\sigma(l)$ has density 0 . The statement and proof of the following proposition are due to the referee.

Proposition 3. Let $M(x)$ denote the number of multiamicable pairs $m, n$ with $m<n$ and $m \leq x$. Then $M(x)=o(x)$ as $x \rightarrow \infty$.

Besides Erdős's lemma, above, we shall also need the following. 
Lemma. For each number $x \geq 1$, we have

$$
\sum_{t \leq x} \frac{\sigma(t)^{2}}{t^{2}}<c x
$$

where $c=\zeta(2)^{2} \zeta(3)$ and $\zeta$ is Riemann's zeta function.

Proof of the Lemma. We have $\sigma(t) / t=\sum_{u \mid t} 1 / u$, so that

$$
\sum_{t \leq x} \frac{\sigma(t)^{2}}{t^{2}}=\sum_{t \leq x} \sum_{u|t, v| t} \frac{1}{u v}=\sum_{u \leq x, v \leq x} \frac{1}{u v}\left[\frac{x}{[u, v]}\right],
$$

where $[u, v]$ is the least common multiple of $u$ and $v$. Hence

$$
\begin{aligned}
\frac{1}{x} \sum_{t \leq x} \frac{\sigma(t)^{2}}{t^{2}} & \leq \sum_{u \leq x, v \leq x} \frac{1}{u v[u, v]}<\sum_{u, v} \frac{\operatorname{gcd}(u, v)}{u^{2} v^{2}}=\sum_{d} \sum_{\operatorname{gcd}(u, v)=d} \frac{d}{u^{2} v^{2}} \\
& <\sum_{d} \sum_{u^{\prime}, v^{\prime}} \frac{d}{\left(d u^{\prime}\right)^{2}\left(d v^{\prime}\right)^{2}}=\sum_{d} \frac{1}{d^{3}} \sum_{u^{\prime}, v^{\prime}} \frac{1}{u^{\prime 2} v^{\prime 2}},
\end{aligned}
$$

which is $\zeta(3) \zeta(2)^{2}$.

Proof of Proposition 3. By Erdős's result, we may restrict our attention to proper multiamicable pairs, since

$$
A(x)=o(x)
$$

if $A(x)$ is the number of amicable pairs $m, n$ with $m<n$ and $m \leq x$. Let $K$ be a large number and let $M_{1}(x)$ denote the number of proper multiamicable pairs $m, n$ with $m \leq x$ and $\sigma(m) / m \geq K$. By (3), if a pair $m, n$ is $(\alpha, \beta)$ amicable, then $\alpha<\sigma(m) / m$, so that the number of $n$ 's that can correspond to a given value of $m$ is less than $\sigma(m) / m$. Thus, by the Lemma,

$$
M_{1}(x) \leq \sum_{m \leq x, \sigma(m) / m \geq K} \frac{\sigma(m)}{m} \leq \frac{1}{K} \sum_{m \leq x} \frac{\sigma(m)^{2}}{m^{2}}<\frac{c}{K} x .
$$

Let $M_{2}(x)$ denote the number of proper multiamicable pairs $m, n$ with $m \leq x, \sigma(m) / m<K$ and $\sigma(n) / n \geq K^{3}$. If such a pair $m, n$ is $(\alpha, \beta)$ amicable, then $n \leq \alpha n=\sigma(m)-m<K m \leq K x$, so that $n<K x$ and $m>n / K$. It follows that $\beta=(\sigma(n)-n) / m<\sigma(n) / m<K \sigma(n) / n$, so that the number of $m$ 's that can correspond to a given value of $n$ is less than $K \sigma(n) / n$. Thus,

$$
M_{2}(x) \leq \sum_{n<K x, \sigma(n) / n \geq K^{3}} \frac{K \sigma(n)}{n} \leq \frac{1}{K^{2}} \sum_{n<K x} \frac{\sigma(n)^{2}}{n^{2}}<\frac{c}{K} x,
$$

by the Lemma.

Finally, let $M_{3}(x)$ denote the number of proper multiamicable pairs $m$, $n$ with $m \leq x, \sigma(m) / m<K$ and $\sigma(n) / n<K^{3}$. As above, if such a pair $m, n$ is $(\alpha, \beta)$-amicable, then $n<K x, m>n / K, \alpha<\sigma(m) / m<K$ and $\beta<\sigma(n) / m<K^{4}$. Let $k$ be the smallest integer such that $L=\alpha^{k} \beta^{k}>K^{7}$ (recalling that $\alpha \beta>1)$. We consider all $(\alpha, \beta)$-amicable pairs $m, n$ that are counted in $M_{3}(x)$, for these values of $\alpha$ and $\beta$. The number of $m$ 's such 
that $m \leq x$ and $L \nmid \sigma(m)$ is $o(x)$, according to Erdös's lemma. Similarly, the number of $n$ 's such that $n<K x$ and $L \nmid \sigma(n)$ is $o(x)$. For the remaining $(\alpha, \beta)$-amicable pairs $m, n$ which are counted in $M_{3}(x)$, we have $L \mid \sigma(m)$ and $L \mid \sigma(n)$. For such a pair, $\alpha n \equiv-m(\bmod L)$ and $\beta m \equiv-n(\bmod L)$ so that $(\alpha \beta-1) m \equiv 0(\bmod L)$. It follows that $L \mid m$. The number of $m$ 's such that $m \leq x$ and $L \mid m$ does not exceed $x / L$. Further, $L>K^{7}$ and there are at most $K(\alpha, \beta)$-amicable pairs containing each such $m$. Thus the number of pairs counted in $M_{3}(x)$ that correspond to these values of $\alpha$ and $\beta$ is at most $x / K^{6}$. Now we consider all multiamicable pairs $m, n$ counted in $M_{3}(x)$. If such a pair is $(\alpha, \beta)$-amicable, then $\alpha<K$ and $\beta<K^{4}$, so that the number of different pairs $\alpha, \beta$ for which we may argue as above is bounded by $K^{5}$. Therefore,

$$
M_{3}(x) \leq \frac{1}{K} x+o(x)
$$

Since $M(x)=A(x)+M_{1}(x)+M_{2}(x)+M_{3}(x)$, and since $K$ can be taken arbitrarily large, Proposition 3 follows from (7), (8), (9) and (10).

We mention finally that we have considered analogous questions for unitary and infinitary divisors, and the results may be obtained from the first-named author.

\section{BIBLIOGRAPHY}

1. S. Battiato and W. Borho, Are there odd amicable numbers not divisible by three?, Math. Comp. 50 (1988), 633-637.

2. R. D. Carmichael, Review of History of the theory of numbers, Amer. Math. Monthly 26 (1919), 396-403.

3. Graeme L. Cohen, On an integer's infinitary divisors, Math. Comp. 54 (1990), 395-411.

4. __ Generalisations of amicable numbers, Internal Report No. 36, School of Mathematical Sciences, University of Technology, Sydney, April 1992.

5. Henri Cohen, On amicable and sociable numbers, Math. Comp. 24 (1970), 423-429.

6. L. E. Dickson, Amicable number triples, Amer. Math. Monthly 20 (1913), 84-91.

7. Leonard Eugene Dickson, History of the theory of numbers, Vol. 1, Chelsea, New York, 1971.

8. P. Erdös, On amicable numbers, Publ. Math. Debrecen 4 (1955), 108-111.

9. Achim Flammenkamp, New sociable numbers, Math. Comp. 56 (1991), 871-873.

10. Richard K. Guy, Unsolved problems in number theory, Springer-Verlag, New York, 1981.

11. Peter Hagis, Jr, Unitary amicable numbers, Math. Comp. 25 (1971), 915-918.

12. C. Krishnamurthy, Some sets of amicable numbers of higher order, Indian J. Pure Appl. Math. 11(12) (1980), 1549-1553.

13. A. Makowski, On some equations involving functions $\phi(n)$ and $\sigma(n)$, Amer. Math. Monthly 67 (1960), 668-670; correction, ibid. 68 (1961), 650.

14. Thomas E. Mason, On amicable numbers and their generalizations, Amer. Math. Monthly 28 (1921), 195-200.

15. Wayne L. McDaniel, On odd multiply perfect numbers, Boll. Un. Mat. Ital. 2 (1970), 185-190.

16. David Moews and Paul C. Moews, A search for aliquot cycles below $10^{10}$, Math. Comp. 57 (1991), 849-855.

17. Paul Poulet, La chasse aux nombres, Bruxelles, 1929. 
18. Herman J. J. te Riele, On generating new amicable pairs from given amicable pairs, Math. Comp. 42 (1984), 219-223.

19. W. Sierpiñski, Elementary theory of numbers, North-Holland, Amsterdam, 1988.

20. Benjamin Franklin Yanney, Another definition of amicable numbers and some of their relations to Dickson's amicables, Amer. Math. Monthly 30 (1923), 311-315.

School of Mathematical Sciences, University of Technology, Sydney, P.O. Box 123 , BroadWay, NSW 2007, Australia

E-mail address: glc@maths.uts.edu.au

Care of School of Mathematical Sciences, University of Technology, Sydney, P.O. Box 123, Broadway, NSW 2007, Australia

Department of Mathematics, Temple University, Philadelphia, Pennsylvania 19122 